\title{
regunaridades en la influencia ejercida por los agentes mineralizadores sobre el proceso de fabricación del clínker de cemento portland blanco
}

\author{
I. F. PONOMAREW, A. N. GRATSCHJAN Y A. P. SUBECHIN \\ Silikattechnik, n. I, enero 1966, pägs. 12-14
}

Para intensificar la cocción de mezclas crudes. que son difíciles de sintetizar al fabricar cementos especiales, hay que emplear adiciones mineralizadoras. Algunos autores (1) y (2) realizaron ensayos para llegar a conocer la relación entre el grado de eficacia de las adiciones mineralizadoras y los cationes presentes en ellas. W. F. Shurawljew (3) demostró que el grado de eficacia de los mineralizadores - sobre todo de los fluoruros- depende tanto de los cationes como de los aniones del mismo. Esto también lo confirman otros autores (4). Sin embargo, no se han encontrado regularidades en el rendimiento. La aclaración de esta cuestión facilitaría la posibilidad de elegir con base científica el mineralizador óptimo para intensificar el proceso de cocción del clínker de cemento.

\section{FINALIDAD Y PROCEDIMIENTO DEL ENSAYO}

Los autores del presente artículo tratan de averiguar la relación entre el grado de eficacia de los mineralizadores y los cationes y aniones presentes en los mismos.

En este trabajo se analizó la influencia mineralizante de cationes de los grupos I y II del sistema periódico de los elementos $-\mathrm{Li}^{+}, \mathrm{Na}^{+}, \mathrm{K}^{+}, \mathrm{Be}^{2+}$ y $\mathrm{Mg}^{2+}-$ y algunos aniones $-\mathrm{SiF}_{6}{ }^{2-}, \mathrm{F}^{-}, \mathrm{SO}_{4}{ }^{2-}$ y $\mathrm{Cl}^{-}$- , que están contenidos en los mineralizadores más eficaces. $\mathrm{Pa}$ ra eliminar la influencia de los aniones al examinar el efecto mineralizante de los cationes se emplearon carbonatos. El análisis se realizó con la mezcla cruda que sinteriza con mayor dificultad y que es característica en la producción de clínker de cemento portland blanco. Tiene un grado de saturación de 0,88 y un módulo de silicatos de 3,5. Los mineralizadores se añaden en forma de sales químicamente puras en cantidades de 0,005, 0,010 y 0,015 equivalentes-gramo por cada $100 \mathrm{~g}$ de mezcla seca.

Se clinkerizaron las mezclas crudas en un horno de laboratorio. Se mantuvo una marcha uniforme al elevar y descender la temperatura de las muestras: elevación de la temperatura a $1.460^{\circ} \mathrm{C}$ en el tiempo de $6 \mathrm{~h}$. A continuación se mantuvo durante $3 \mathrm{~h}$ esta misma temperatura. El enfriamiento del clínker se realizó en agua. 


\section{INFLUENCIA DE LOS CATIONES}

El análisis de la fijación de cal demuestra (fig. 1) que con temperaturas de cocción por encima de $1.150^{\circ} \mathrm{C}$ la influencia favorable de los cationes en el proceso de formación del clínker disminuye en el siguiente orden:

$$
\mathrm{Be}^{2+} \longrightarrow \mathrm{Mg}^{2+} \longrightarrow \mathrm{Li}^{+} \longrightarrow \mathrm{Na}^{+} \longrightarrow \mathrm{K}^{+} .
$$

$\mathrm{El}$ análisis por rayos $\mathrm{X}$ de la composición de fases de los productos sinterizados tratados

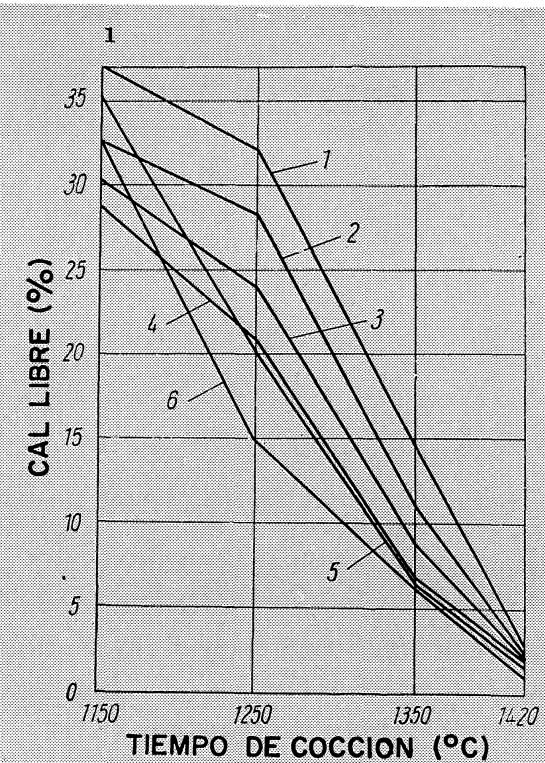

Fig. 1.-Fijación de cal durante la cocción del erudo. 1. - Sin adición; 2-6.-Con adición de 0.015 equivalentes gramo de carbonato 100 a de mezcla $(2, \mathrm{CO} \mathrm{K}$ $/ 100 \mathrm{~g}$ de mezcla $\left(2-\mathrm{CO}_{3} \mathrm{~K}_{2}\right.$

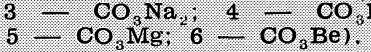
a diferentes temperaturas y los análisis termográficos del proceso de cocción, confirmaron plenamente el orden de los cationes en esta serie.

El grado de eficacia variable de los mineralizadores puede explicarse a la luz de las observaciones más modernas (5) y (6), teniendo en cuenta las características electrostáticas diferentes de los cationes y aniones presentes en los mineralizadores.

Una comparación de la influencia mineralizante de los cationes con tamaños diferentes definidos por su posición en el sistema periódico, muestra que la influencia da los cationes sobre el proceso de formación del clínker depende de su electronegatividad (7): al disminuir la electronegatividad del catión, también disminuye el grado de eficacia de la influencia mineralizante (tabla 1 ).

T A B L A 1

Relación entre el poder mineralizador de los cationes y su electronegatividad

\begin{tabular}{lll}
\hline Elementos & $\begin{array}{c}\text { Electronegatividad } \\
\text { de los } \\
\text { elementos }\end{array}$ & $\begin{array}{c}\text { Disminución del efecto } \\
\text { mineralizador } \\
\text { de los cationes }\end{array}$ \\
$\mathrm{Be}$ & 1,5 & $\mathrm{Be}^{2+}$ \\
$\mathrm{Mg}$ & 1,2 & $\mathrm{Mg}^{2+}$ \\
$\mathrm{Li}$ & 1,0 & $\mathrm{Li}^{+}$ \\
$\mathrm{Na}$ & 0,9 & $\mathrm{Na}^{+}$ \\
$\mathrm{K}$ & 0,8 & $\mathrm{~K}^{+}$ \\
\hline
\end{tabular}


Los análisis de la microestructura del clínker permitieron observar que el carácter de la influencia de los cationes es distinto según su electronegatividad. Los cationes con gran electronegatividad $\left(\mathrm{Be}^{2+}, \mathrm{Mg}^{2+}, \mathrm{Li}^{+}\right)$producen un crecimiento máximo de los cristales de los constituyentes del clínker y crean una estructura con cristales grandes. La forma de los cristales está casi exenta de defectos. Los cationes son electronegatividad reducida $\left(\mathrm{Na}^{+}\right.$, $\mathrm{K}^{+}$) fomentan la formación de una estructura en el clínker con cristales finos. Los tamaños de los cristales se encuentran entre 5 y $10 \mu \mathrm{m}$, y la forma de los cristales es defectuosa. Esas diferencias en la estructura del clínker son de gran importancia, sobre todo en la fabricación de cemento portland blanco.

Como mostraron las determinaciones del contenido en blanco, la estructura cristalina gruesa del clínker, al introducir mineralizadores con los cationes $\mathrm{Be}^{2+}, \mathrm{Mg}^{2+}$ y $\mathrm{Li}^{+}$, conduce a una disminución del contenido en blanco del clínker de un 3 a 4 por ciento.

\section{INFLUENCIA DE LOS ANIONES}

Los autores analizaron la eficacia mineralizante de los aniones $\mathrm{SiF}_{6}{ }^{2-}, \mathrm{F}^{-}, \mathrm{SO}_{4}{ }^{2-}$ y Cl$^{-}$al introducirlos en un crudo con el catión $\mathrm{Na}^{+}$. Como se viene a comprobar en el trabajo, este catión tiene la influencia más favorable sobre la estructura y el contenido en blanco del clínker de cemento portland blanco. Además se analizaron sales de calcio con los aniones mencionados, siendo éstos los mineralizadores más frecuentes en la práctica industrial.

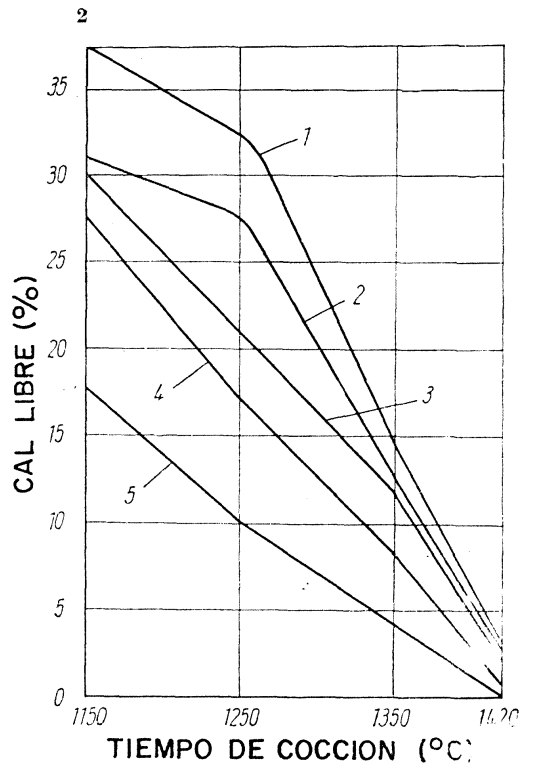

Fig. 2.-Fijacion de cal durante la cocción del crudo. 1.-Sin adición; 2-5.-Con adición de 0,015 equivalentes gramo de carbonato/ $/ 100 \mathrm{~g}$ de mezcla $(2-\mathrm{ClNa}$; 3 - $\mathrm{SO}_{4} \mathrm{Na}_{2} ; 4$ - $\mathrm{FNa} ; 5$ $-\mathrm{SiF}_{6} \mathrm{Na}_{2}$ ).
Los resultados obtenidos al estudiar la fijación de cal (fig. 2), muestran que la adición del anión $\mathrm{SiF}_{6}{ }^{2-}$ en cooperación con el catión $\mathrm{Na}^{+}$consigue la mayor eficacia mineralizante. La eficacia es un poco menor con una adición anión $\mathrm{F}^{-}$. La eficacia mineralizante de los aniones $\mathrm{SO}_{4}{ }^{2-}$ y $\mathrm{Cl}^{-}$es considerablemente menor: incluso con una dosificación máxima $-0,015 \mathrm{~g}$ equivalentes-gramo de mineralizadores con estos aniones en $100 \mathrm{~g}$ de mezclaqueda en el clínker una cantidad elevada de $\mathrm{CaO}$ no absorbida.

Como muestran las observaciones termográficas y con rayos $\mathrm{X}$ del proceso de formación del clínker, la influencia de los aniones de los mineralizadores sobre la formación del clínker aparece en el siguiente orden decreciente:

$$
\mathrm{SiF}_{6}{ }^{2-} \longrightarrow \mathrm{F}^{-} \longrightarrow \mathrm{SO}_{4}{ }^{2-} \longrightarrow \mathrm{Cl}^{-} .
$$

Al comparar la eficacia mineralizante de los aniones con su electronegatividad (tabla 2), también se demuestra que al disminuir la electronegatividad disminuye su eficacia mineralizante. 
T A B L A 2

Relación entre el poder mineralizador de los aniones y su electronegatividad

\begin{tabular}{lll}
\hline Aniones & $\begin{array}{c}\text { Electronegatividad } \\
\text { de los } \\
\text { aniones }\end{array}$ & $\begin{array}{c}\text { Disminución del poder } \\
\text { mineralizador } \\
\text { de los aniones }\end{array}$ \\
\hline $\mathrm{SiF}_{6}{ }^{2-}$ & 3,9 & $\mathrm{SiF}_{6}{ }^{2-}$ \\
$\mathrm{F}^{-}$ & 3,6 & $\mathrm{~F}^{-}$ \\
$\mathrm{SO}_{4}{ }^{2-}$ & 3,1 & $\mathrm{SO}_{4}{ }^{2-}$ \\
$\mathrm{Cl}^{-}$ & $\mathrm{Cl}^{-}$ \\
\hline
\end{tabular}

\section{INFLUENCIA CONJUNTA DE CATIONES Y ANIONES}

Cuando se colocan los aniones analizados con el catión $\mathrm{Ca}^{2+}$ en la mezcla cruda, el orden en que disminuye su eficacia mineralizante es el mismo que con el catión $\mathrm{Na}^{+}$. Sin embargo, el grado de eficacia de estos aniones junto con el catión $\mathrm{Ca}^{2+}$ es considerablemente más bajo que al emplear el catión $\mathrm{Na}^{+}$. Este fenómeno se explica por el hecho de que al cooperar el anión con un catión de mayor electronegatividad, disminuye la diferencia absoluta entre los valores numéricos de su electronegatividad, lo que puede considerarse como una medida de la energía del mineralizador (6). De esta forma, los mineralizadores que se componen de un anión con mayor electronegatividad y de un catión con mínima electronegatividad tienen el mayor grado de eficacia.

Esta regularidad hallada por los autores se comprueba plenamente con los resultados de ensayos encontrados por otros autores (4), que se recibieron al analizar clínkeres de diferente composición mineralógica.

Al observar microestructuras se averiguó que, al emplear diferentes aniones, aumenta el tamaño de los cristales de los minerales del clínker al disminuir la electronegatividad de aquéllos. Así, al emplear juntos los aniones y el catión $\mathrm{Ca}^{2+}$, se crea una estructura de los cristales del clínker más gruesa que al emplear $\mathrm{Na}^{+}$. Esto se explica por el hecho de que el catión $\mathrm{Ca}^{2+}$, como centro de cristalización de los minerales, fomenta más el crecimiento de los cristales. Esta comprobación coincide con las averiguaciones hechas por otros autores (8 a 10$)$.

\section{LA INFLUENCIA DE CATIONES $Y$ ANIONES SOBRE LA FASE LIQUIDA DEL CLINKER}

Puesto que el proceso de formación, la estructura y las propiedades del clínker vienen determinados en gran medida por la composición y por las propiedades de su fase líquida (11 a 14), los autores estudiaron la influencia de los cationes y aniones, antes mencionados, en la citada fase líquida del clínker.

Los análisis con rayos $\mathrm{X}$ de las muestras obtenidas permiten comprobar que, al aumentar la electronegatividad de los cationes y aniones, disminuye la cantidad de fase cristalina en el clínker. Esto es consecuencia de una disminución considerable en la viscosidad de la fase líquida del clínker al emplear cationes y aniones con gran electronegatividad. 
Los análisis magnetoquímicos de muestras de la fase líquida del clínker señalaron una influencia considerable de los mineralizadores en función de la electronegatividad de sus cationes y aniones, que condujo a una modificación de la susceptibilidad magnética específica de las muestras. La modificación en la susceptibilidad magnética de la fase líquida del clínker, denota modificaciones cristaloquímicas que se producen en fracción ferruginosa de dicha fase líquida. Las comprobaciones a base de la resonancia paramagnética y mecanocuántica muestran que los cationes y aniones de los mineralizadores conducen a una modificación en el número de coordinación de los iones del hierro trivalente, que es una de las causas en la disminución del contenido en blanco del clínker al emplear algunos mineralizadores. En consecuencia, los mineralizadores, según la electronegatividad de los cationes y aniones existentes en los mismos, influyen de manera decisiva en la fase líquida del clínker y, por ello, influyen en la formación de minerales, en la cristalización y en la estructura del clínker.

\section{O N C L U S I O N E S}

Los resultados de los ensayos realizados justifican la afirmación de que los materiales más eficaces son aquellos mineralizadores en los que existe un anión con la mayor y un catión con la menor electronegatividad. Uno de los mineralizadores es el hexafluosilicato sódico, que reúne de manera óptima el anión $\mathrm{SiF}_{6}{ }^{2-}$ (de gran electronegatividad) y el catión $\mathrm{Na}^{*}$ (de pequeña electronegatividad). El hexafluosilicato sódico provoca además la formación de una estructura fina del clínker, con lo cual aumenta el contenido en blanco al fabricar cemento portland blanco.

\section{B I B L I O G R A F I A}

(1) Awgustinnik, A. I., und Kurdewanidse, O. K.: Shurnal prikladnoi chimii 20 (1947) Nr. 12.

(2) Sytschew, M. M., Astachow, M. A., und Konoвowa, W. J.: Iswestija Wusow "Chimija $i$ chimitscheskaja technologija" 2 (1959) 5, S. 755 - 760.

(3) ShurawlJEw, W. F.: Trudy 5-go sowestschanija sawodskich laboratorii zementnoi promyschlennosti (Arbeiten der 5. Konferenz der Betriebslaboratorien der Zementindustrie). 1950.

(4) Okorokow, S. D., Golynko-Wolfson, S. L., SchewelJowa, B. I., und Jarkina, T. N.: Zement, Leningrad (1957) 3 , S. $5-11$.

(5) Wolkonski, B. W., Konowalow, P. F., und Makaschew, S. D.: Mineralisatory w zementnoi promyschlennosti (Mineralisatoren in der Zementindustrie). Stroiisdat, Moskau 1964.

(6) Ertel, W.: Physikalische Chemie der Silikate (russ.). Isdatelstwo IL, Moskau 1962.

(7) BAzANow, S. S.: Elektrootrizatelnost elementow i chimitscheskaja swjas (Elektronegativität der Elemente und chemische Bindung). Sib. otd. Akad. Nauk SSSR, 1962.

(8) Jessin, O. A.: Isw. Akad. Nauk SSSR, Otdelenije chim. nauk, Mokau (1948) Nr. 6.

(9) Wolkonski, B. W., und Haschkowskaja, A. P.: Trud. "Giprozement" 1961, Folge 23.

(10) Malkin, W. I., und Mogutnow, B. M.: Arb. d. Unionskonf. über die physikalische Chemie der Schmelzflüsse und Schlacken (russ.). Metallurgisdat, Moskau 1962.

(11) Toropow, N. A.: Chimija zementow (Zementchemie). Promstroiisdat, Moskau 1956.

(12) Butt, J. M., und Schachmagon, N. W.: Issledowanije mechanisma mineralisujustschego deistwija kremneftoristogo natrija (Untersuchung des Mechanismus der mineralisierenden Wirkung des Natriumhexafluoroilikat). Arbeiten des Instituts "NIIZement", Moskau 1961, Folge 15.

(13) Jizku, E.: Stavivo 36 (1958) 3, S. $91-96$.

(14) Vogel, E.: Silikattechnik 10 (1959) 8, S. $415-419$. 